\title{
Cold Spray Deposition of Thermoelectric Materials
}

\author{
ALEXANDER A. BAKER (iD, ${ }^{1,4}$ RICHARD THUSS, ${ }^{2}$ NATHAN WOOLLETT,${ }^{1}$ \\ ALYSSA MAICH, ${ }^{1,3}$ ELISSAIOS STAVROU $\odot,{ }^{1}$ SCOTT K. MCCALL, ${ }^{1}$ \\ and HARRY B. RADOUSKY ${ }^{1}$ \\ 1.-Lawrence Livermore National Laboratory, 7000 East Avenue, Livermore, CA 94550, USA. \\ 2.-TTEC Thermoelectric Technologies, Wickliffe Road, Berryville, VA 22611, USA. \\ 3.-University of the Pacific, 3601 Pacific Ave, Stockton, CA 95211, USA. \\ 4.-e-mail: baker97@llnl.gov
}

Thermoelectric materials convert heat flux to electricity (or vice versa as Peltier coolers); however, their application to harvest waste heat is limited by challenges in fabrication and materials optimization. Here, cold-spray deposition is used as an additive manufacturing technique to fabricate $p$ - and $n$ type $\mathrm{Bi}_{2} \mathrm{Te}_{3}$, on substrates ranging from quartz to aluminum. The sprayed material has a randomly oriented microstructure largely free from pores ( $>99.5 \%$ dense), and deposition is achieved without substantial compositional changes. The Seebeck coefficient and thermal conductivity are largely preserved through the spray process, but the defects introduced during deposition significantly increase electrical resistivity. Defects can be removed, and compressive strain relaxed by a post-deposition anneal, which leads to $\mathrm{Bi}_{2} \mathrm{Te}_{3}$ blocks with a typical $Z T$ of 0.3 at $100^{\circ} \mathrm{C}$. Generators fabricated on sheets or pipes made of copper compare favorably with similar designs constructed using bulk $\mathrm{Bi}_{2} \mathrm{Te}_{3}$, displaying a wider operating temperature range. These results demonstrate the power and versatility of cold-spray additive manufacturing and provide a pathway toward fabrication of thermoelectric generators in complex geometries that are inaccessible to generators made by traditional approaches.

\section{INTRODUCTION}

Cold-spray (CS) deposition of coatings is widely used across industry for corrosion-resistant claddings, surface functionalization and localized repair. ${ }^{1-4}$ In this technique, micron-scale metal particles are entrained in supersonic gas (typically $\mathrm{He}$ or $\mathrm{N}_{2}$ ) and directed onto a metal surface. Upon impact, the particles plastically deform and bond with the surface or one another, ${ }^{5}$ with deposition efficiency controlled by particle speed and ductility ${ }^{6}$-although the precise mechanism remains the subject of some debate. ${ }^{7,8}$ Due to the requirement for plastic deformation, CS has typically been limited to malleable materials, making it well suited for structural elements and alloys, ${ }^{7}$ but unsuited for functional materials, which are typically brittle semiconductors or insulators. Nevertheless, as cold spray operates at comparatively low temperatures, beneath the melting point of most functional materials, it is attractive to consider the possibility of an additive manufacturing technique that thus preserves the tailored microstructure that drives functional properties. ${ }^{9-11}$

One such material class is thermoelectrics, wherein a thermal gradient creates an electric potential, and vice versa. ${ }^{12}$ Thermoelectric generators (TEGs) couple $p$ - and $n$-type thermoelectrics to generate an electric current, converting waste heat into useful electricity. ${ }^{13}$ With US industry alone rejecting some quadrillions of BTUs annually, ${ }^{14}$ there is significant appetite for a technology that can economically recoup a portion of this loss. Furthermore, as TEGs have no moving parts and are not based on chemical reactions, they have a long operating life with no maintenance requirements, making them excellent candidates for power sources in remote or inaccessible locations. ${ }^{15}$ To date, adoption of TEGs to harvest waste heat has been limited, in part because of the difficulty of fabricating parts that efficiently absorb heat being vented from cooling fins or radiated from transfer 
pipes. From a simple geometric argument, it would require six planar TEGs to capture $90 \%$ of the heat that can be harvested with a single curved TEG mounted on the outside of a pipe. It is thus desirable to find an approach that can fabricate TEGs in place on curved or more complex geometries, without the need for expensive cutting and dicing steps, and without loss of material properties. Laser melting and consolidation as well as plasma spray have been considered solutions to this problem, ${ }^{11,16}$ although porosity and lack of microstructural control may prove to be limiting factors. ${ }^{17}$

Here, we demonstrate successful fabrication of TEGs using cold-spray additive manufacturing of bismuth telluride powders. Layers of $p$ - and $n$-type thermoelectric can be deposited on a wide range of substrates, from stainless steel to aluminum silicate and quartz, on flat and curved geometries. The deposited bismuth telluride is composed of randomly oriented polycrystals, with comparable Seebeck and thermal conductivity values to the starting bulk material, but an elevated resistivity. Thermal annealing at $400^{\circ} \mathrm{C}$ relaxes the compressive strain introduced in deposition and removes other defects, reducing resistivity, thereby enhancing $Z T$. TEGs fabricated in place on flat $\mathrm{Cu}$ surfaces or pipes yield comparable current and voltage values to those produced by bulk materials mounted on the same surface, demonstrating the viability of this technique for industrial manufacturing applications.

\section{EXPERIMENTAL}

Several bulk $n$ - and $p$-type $\mathrm{Bi}_{2} \mathrm{Te}_{3}$ formulations were used in the study, including billets from Custom Thermoelectric Corp. (Bishopville, MD, USA) and Everredtronics Ltd. (Shanghai, China). Each billet contained Se and Sb additives to control the carrier concentration, but are hereafter referred to as $\mathrm{Bi}_{2} \mathrm{Te}_{3}$ for simplicity. Feedstock powders were prepared from bulk crystals by multiple cycles of high energy ball milling, using alumina balls and crucibles, to achieve polydisperse particles with diameter $<10$ microns. The powders were sieved to remove larger agglomerates prior to deposition to reduce the risk of nozzle clogging during the process. After this, the particles were stored in an oven at $60^{\circ} \mathrm{C}$ for $3 \mathrm{~h}$ to remove any atmospheric moisture. The cold-spray deposition was performed using a TTEC LLC, custom-designed, type-one, lowpressure cold spray system (Fig. 1). The nozzle design parameters and nitrogen gas temperature and gas pressure conditions were optimized to achieve the best results for spraying $\mathrm{Bi}_{2} \mathrm{Te}_{3}$ formulations with particle velocities of $850-900 \mathrm{~m} / \mathrm{s}$. A type-one system uses the expansion of the gas in the diverging section of the nozzle to create a suction that draws the $\mathrm{Bi}_{2} \mathrm{Te}_{3}$ powder into the supersonic gas stream. ${ }^{18}$ The as-milled $\mathrm{Bi}_{2} \mathrm{Te}_{3}$ powders are highly cohesive and resistant to flow. A flow additive was used for some of the deposition tests to control the rate of powder flow into the nozzle; extensive testing has shown that this additive is not present in the final deposited material.

Synchrotron angle-dispersive x-ray diffraction measurements were performed using a MAR-CCD detector at the ALS Beamline 12.2.2 and a Pilatus $1 \mathrm{M} \mathrm{CdTe}$ detector at the undulator Beamline 13ID-D at GeoSoilEnviroCARS, sector13, APS. The xray probing beam spot size was focused to approximately $10 \mu \mathrm{m}$ using Kirkpatrick-Baez mirrors. Integration of powder diffraction patterns to yield scattering intensity versus $2 \theta$ diagrams and initial analysis were performed using the DIOPTAS program. ${ }^{19}$ Calculated XRD patterns were produced using the POWDER CELL program ${ }^{20}$ for the corresponding crystal structures and assuming continuous Debye rings of uniform intensity.

Scanning electron microscopy (SEM) investigations were carried out with a Tescan, Vega3 scanning electron microscope, in secondary electron mode, at an accelerating voltage of $5 \mathrm{kV}$. Glass slides were affixed to the Aluminum SEM pegs with carbon tape.

Electrical and thermal transport (electrical resistivity, $\rho$, thermal conductivity, $\kappa$, and Seebeck coefficient, $S$ ) measurements were performed using the thermal transport option (TTO) of a Quantum Design PPMS in the range $2-400 \mathrm{~K}$. From these parameters, the thermoelectric figure of merit, $Z T$, was calculated using the equation $Z T=S^{2} T / \rho \kappa{ }^{13}$ Samples of approximately $2 \mathrm{~mm} \times 2 \mathrm{~mm} \times 15 \mathrm{~mm}$ dimensions were cut from billets and cold-sprayed blocks using a diamond saw and polished using finegrit sandpaper to obtain uniform cross section and clean surfaces.

The gas dynamics were modeled using the COMSOL 15.4 High Mach Number Flow module with a $k-\varepsilon$ turbulence model. The gas model was solved using the Stationary Solver. The effect of the particles in the gas flow was negligible, allowing for a single solution to the gas dynamics to be used for many particle models. The particle trajectories were modeled by using the COMSOL 15.4 Particle Tracing for Fluid Flow module and the Time Dependent Solver.

\section{RESULTS AND DISCUSSION}

Doped $\mathrm{Bi}_{2} \mathrm{Te}_{3}$ of both $\mathrm{p}$ and $\mathrm{n}$ type has been deposited on a wide variety of substrates to demonstrate the flexibility of the cold-spray technique. Test blocks have been fabricated on copper, aluminum, 316 stainless steel, quartz, glass, aluminum silicate and a syntactic silicon foam, showing impressive versatility (see Fig. 1 for representative examples). No change in deposition efficiency was observed as a function of substrate hardness, conductivity (electrical or thermal) or density. For smooth surfaces, the first few seconds of spray do not lead to any deposition, instead appearing to roughen the surfaces before particles start to embed 

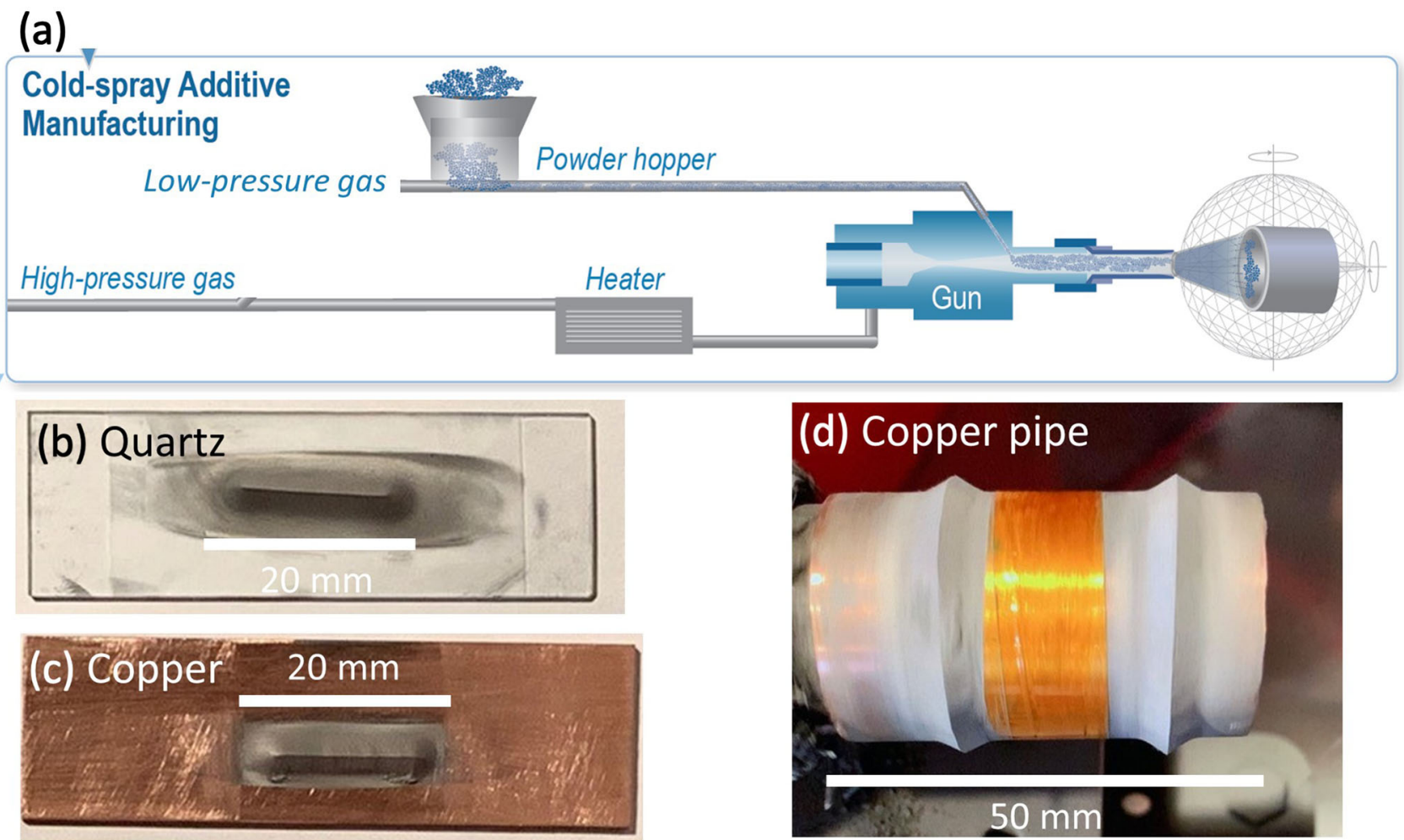

Fig. 1. (a) Schematic of the cold-spray process, showing powder feed and gas handling. Note that the powder is injected after the expansion in the throat of the gun. (b) and (c) Depositions of $\mathrm{Bi}_{2} \mathrm{Te}_{3}$ bars on quartz and copper, respectively; the height of the material is approximately 3 mm. (d) Uniform deposition was achieved over the full surface by rotating the pipe during the spray process for lines of $p$ - and $n$-type $\mathrm{Bi}_{2} \mathrm{Te}_{3}$. $\mathrm{Kapton}$ tape prevented overspray from making direct thermal and electrical contact between the two electrodes.

and interlock. A similar effect can be achieved using a light chemical etch or mechanical abrasion prior to deposition. Helium pycnometry measurements of the density of sprayed $\mathrm{Bi}_{2} \mathrm{Te}_{3}$ compared with the precursor billet showed that the cold-sprayed deposition was $>99.5 \%$ dense.

COMSOL was used to model the gas and particle flow dynamics for a typical deposition on flat and curved surfaces. In both cases, it was shown that the supersonic shock front is located outside of the nozzle, with velocities as high as $900 \mathrm{~m} / \mathrm{s}$ being achieved $5 \mathrm{~mm}$ from the substrate for our given inlet gas pressure and temperature. There is a localized region of reduced velocity at the point of impact, caused by rapid deceleration at the substrate, and associated deflection to the sides. This is visible as jets of high-velocity gas moving perpendicular to the original stream in the case of deposition on flat substrates. It is notable that these jets conform to the surface in the case of curved substrates rather than being deflected at right angles to the point of impact. The resulting gas pressure at the substrate can be as high as $482 \mathrm{kPa}$, which may aid the adhesion process by compacting deposited material. The spray particles were modeled with the appropriate density and radius for the powder characteristics but were modeled as spheres rather than random crystalline shards to simplify calculations. The particles were shown to accelerate rapidly upon injection to the gas stream, achieving velocities comparable to the gas flow and showing minimal deceleration over the shock front. If the particles do not adhere on the first impact, the gas circulation is sufficient to produce secondary impacts for small scattering angles and the removal of loose material for large scattering angles.

Energy-dispersive x-ray spectroscopy (EDS) scans at multiple positions across the sprayed $\mathrm{Bi}_{2} \mathrm{Te}_{3}$ show that elemental composition does not vary across the extent of the sample, confirming that deposition is homogeneous. The elemental composition is $\mathrm{Bi}_{0.5} \mathrm{Sb}_{1.5} \mathrm{Te}_{3.05} \mathrm{Se}_{0.15}$ for $\mathrm{p}$ type and $\mathrm{Bi}_{1} \mathrm{Te}_{0.95} \mathrm{Se}_{0.05}$ for $n$ type. These values are unchanged from the starting billet material, within experimental error. However, it is notable that both deviate significantly from the base composition of $\mathrm{Bi}_{2} \mathrm{Te}_{3}$ because of the addition of $\mathrm{Sb}$ and Se dopants to control the carrier density and type. XRD measurements (Fig. 2e) reveal that the sprayed $\mathrm{Bi}_{2} \mathrm{Te}_{3}$ is a randomly oriented polycrystal, with a crystal structure matching that of the billet. It is also notable that the peaks shift to lower $Q$ after annealing the as-sprayed sample, indicative of an increase in volume of the unit cell and expansion of the lattice. This suggests the presence of compressive strain, introduced during the spraying process, which is relaxed after $10 \mathrm{~h}$ at $400^{\circ} \mathrm{C}$. This need to manage residual stress is common in materials 


\section{(a) Comsol Simulations of Deposition}
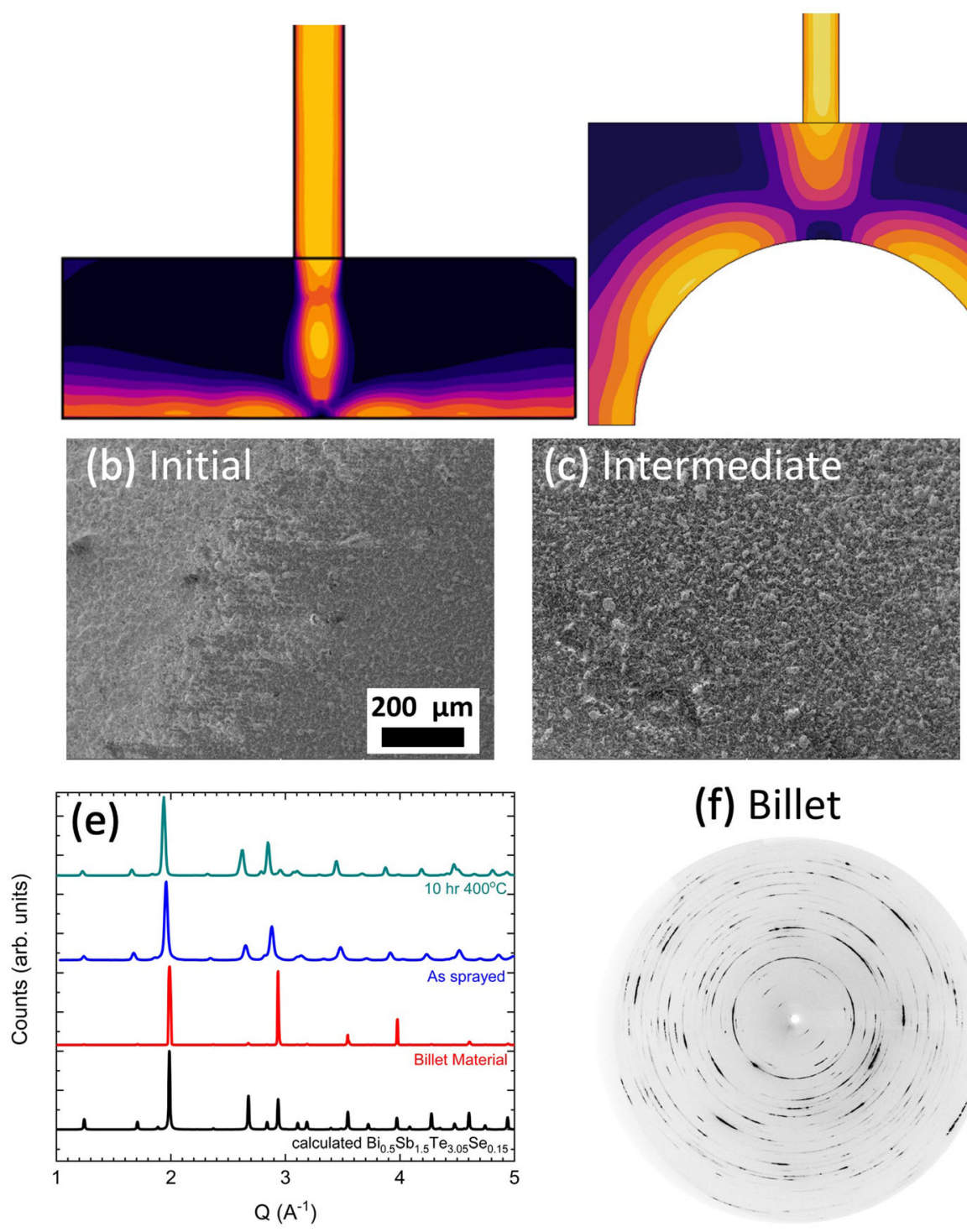

(f) Billet
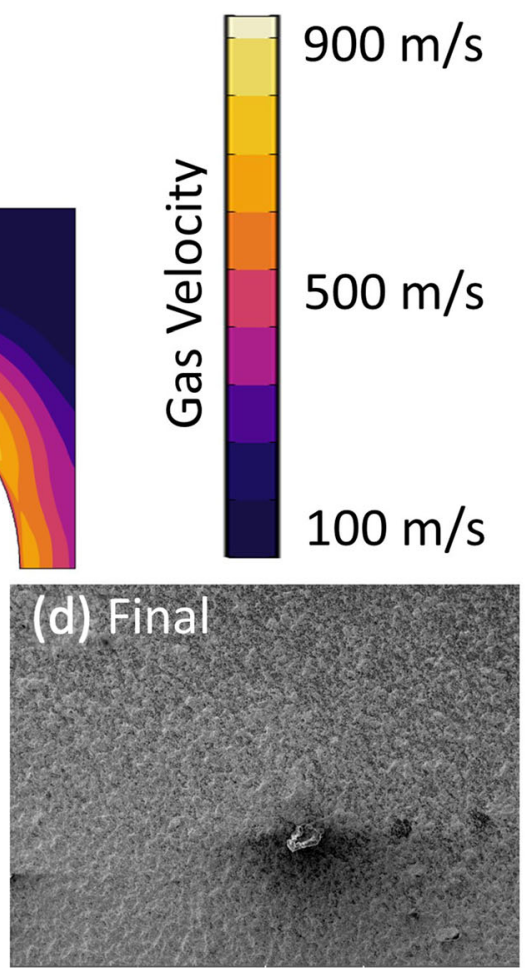

(g) As Sprayed
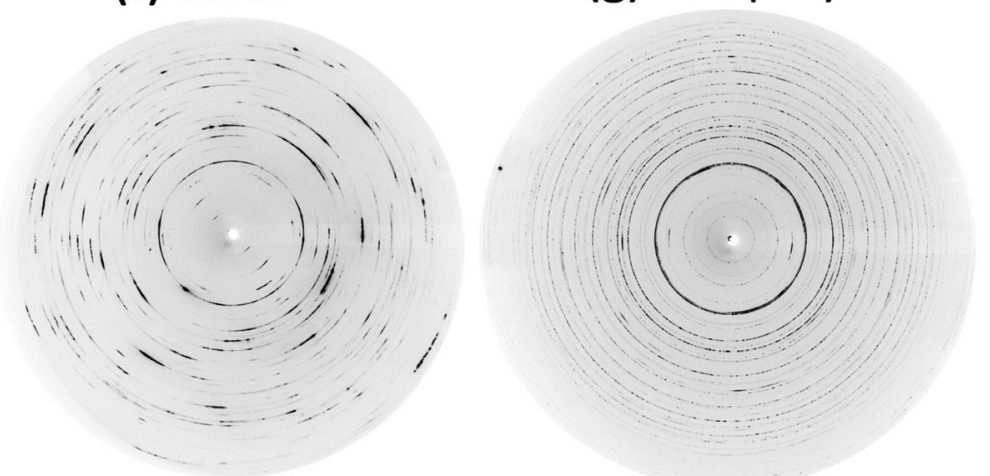

Fig. 2. (a) COMSOL simulation of gas (and thus particle) velocity during deposition on a flat surface, demonstrating that the supersonic shock occurs outside the nozzle, resulting in high gas pressures at the deposition site, which aid particle build-up. (b-d) The buildup process on a quartz substrate [seen in the right of (b)], with micron-scale particles embedding in the substrate to form a base layer, to which subsequent particles interlock and are compacted by subsequent particle impacts. (e) XRD results for $p$-type billet and spray material (before and after annealing) and the calculated pattern for $\mathrm{Bi}_{0.5} \mathrm{Sb}_{1.5} \mathrm{Te}_{3.05} \mathrm{Se}_{0.15}$. There is no evidence of a phase change, but the texture is modified, as seen in in (f) and (g), where $2 \mathrm{D}$ diffraction patterns become uniform rings as a result of the random orientation imposed during deposition.

made by advanced manufacturing methods. ${ }^{21-23}$ The random orientation, coupled with micron-scale crystallites formed during the ball-milling process, leads to the presence of grain boundaries and other defects that can decrease electrical and thermal conductivity.

The mechanism of adhesion and build-up of layers was assessed by performing SEM imaging of the spray onto quartz as a function of deposition time; representative results are shown in Fig. $2 b$, c, and d. Initially, the particles sandblast and roughen the surface; adherence starts once the surface is sufficiently rough to allow mechanical interlocking and embedding of the micron-scale particles. Examining the edge of the sprayed area (as in Fig. 2b) reveals a well-delineated boundary, with a partially covered region $\sim 100$ microns wide. As deposition continues, the initial particles are compressed by subsequent impacts, particularly of larger particles or agglomerates, leading to the high density observed by pycnometry. The rough surface is decorated by the larger particles or loose pieces. Over time, further 
particle impacts smooth and densify the surface, although particles on the order of 50 microns can still be observed (see Fig. 2d lower right corner). The presence of particles much larger than the feedstock powder suggests either aggregation within the gas flow or that pieces of the deposited material can be broken free by subsequent impacts. This mechanism of interlocking and compaction is distinct from the plastic deformation and "splatting" that takes place in conventional metal cold spray, wherein the ductile particles change shape in response to the high speed of the impacts. Examination of deposited particles and overspray revealed no evidence of plastic deformation, rather the brittle $\mathrm{Bi}_{2} \mathrm{Te}_{3}$ is more likely to shatter or break. High-speed imaging of particle impacts would be invaluable in shedding further light on the process.

The measured thermoelectric properties of $n$-type billet and cold-sprayed material are presented in Fig. 3; similar results are observed for $p$ type. There is a modest reduction in the Seebeck coefficient and thermal conductivity. The random orientation and increased defect concentration in sprayed $\mathrm{Bi}_{2} \mathrm{Te}_{3}$ reduce both electron and phonon heat conduction, resulting in a notably different low-temperature behavior. Thermal annealing removes a significant fraction of the defects, demonstrated by the large decrease in resistivity and improvement in low temperature thermal conductivity. The random orientation remains after annealing, and the intrinsic resistivity is about three times larger along the crystalline c-axis than in the ab plane. This increase in resistivity sharply depresses $Z T$ with respect to the starting material. Reducing thermal conductivity while retaining electrical conductivity is an ongoing challenge in thermoelectric material optimization; they are highly correlated properties, as described by the Wiedermann-Franz law. ${ }^{24}$ The values of $Z T$ obtained for the billet material are in reasonable agreement with those previously reported for bulk $\mathrm{Bi}_{2} \mathrm{Te}_{3}{ }^{25,26}$ as well as by commercial suppliers. The decreased $Z T$ of the sprayed material relative to these performance metrics demonstrates that.
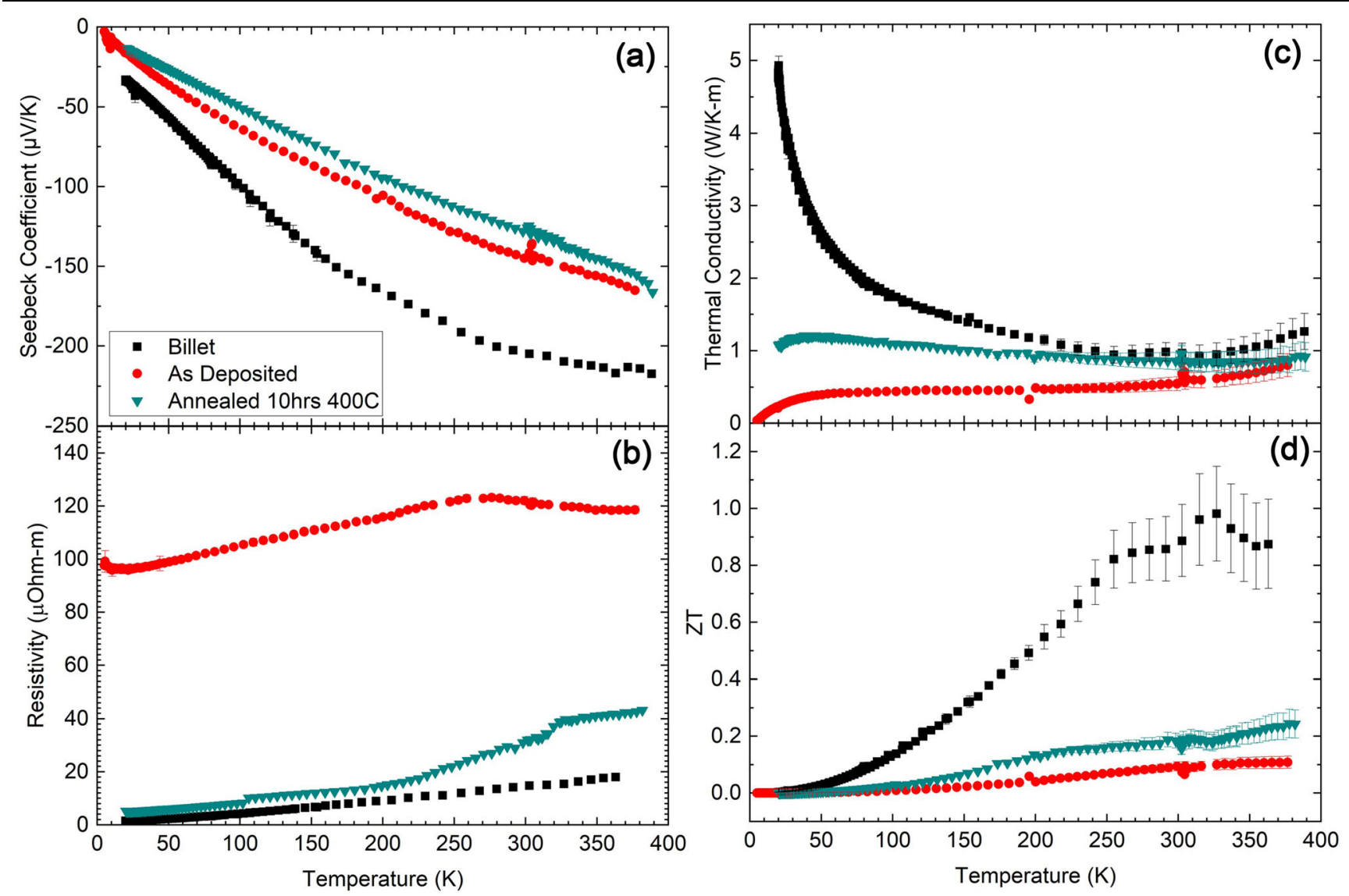

Fig. 3. Thermoelectric properties of $n$-type $\mathrm{Bi}_{2} \mathrm{Te}_{3}$ in the billet (precursor) form, along with cold-sprayed (as deposited) and cold-spray deposition annealed in an Ar atmosphere. Note the Seebeck values are negative. Seebeck and thermal conductivity (a, c) values are similar or slightly reduced from the bulk form above room temperature. Resistivity in the as-deposited state is significantly increased (b), likely because of the presence of defects, leading to a much lower thermoelectric figure of merit, $Z T$ (d). However, annealing reduces resistivity, leading to a final $Z T$ of $\sim 0.3$, which compares favorably with the 0.9 of the billet material. 

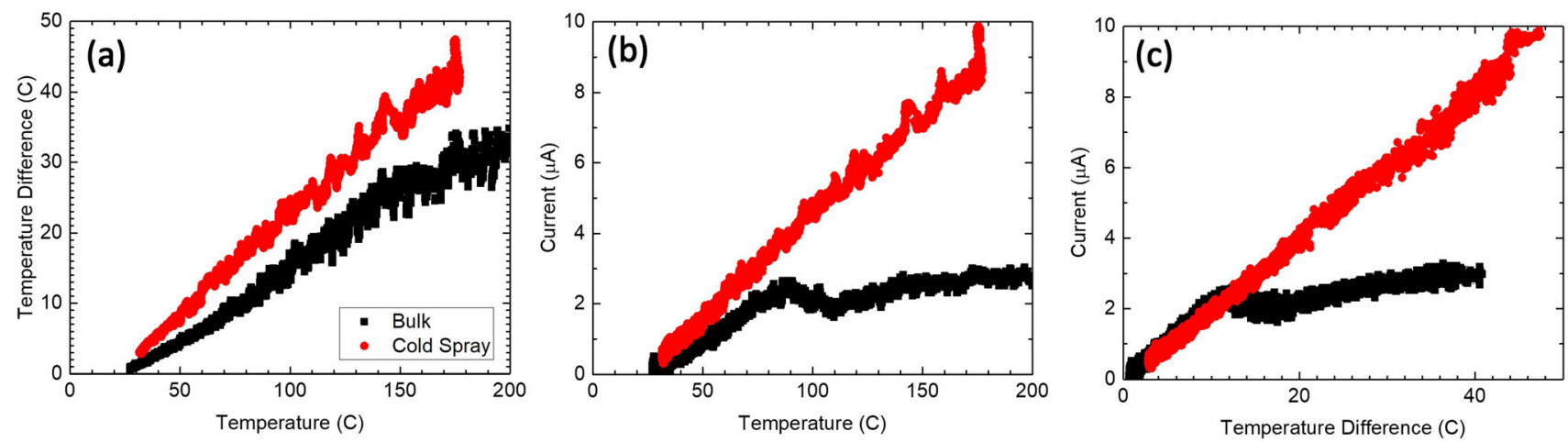

Fig. 4. TEG performance properties for a single couple composed of blocks of bulk $\mathrm{Bi}_{2} \mathrm{Te}_{3}$ (black) and sprayed $\mathrm{Bi}_{2} \mathrm{Te}_{3}(\mathrm{red}$ ), on the surface of a copper pipe uniformly heated from below. The similar thermal conductivity of the two materials leads to similar thermal gradients across the TEGs (as shown in (a), defined as the difference between the temperature at the Cu pipe and the top of the TE elements), which are converted to the currents shown in (b). Notably, the sprayed material demonstrates good performance across the full temperature range, whereas the billet does not improve once the hot side temperature exceeds $100^{\circ} \mathrm{C}$, as expected based on the dropping $Z T$. Data from (b) is replotted in (c) as function of gradient across the TEG element (Color figure online).

Since the cold-spray process naturally introduces many defects, as well as a random orientation of the grains, it should be possible to improve crystal quality with an annealing treatment around the homologous temperature of $\mathrm{Bi}_{2} \mathrm{Te}_{3}$. Several schedules were attempted, with the most promising results coming from a $10-\mathrm{h}$ anneal at $400^{\circ} \mathrm{C}$ in a flowing Ar. The high resistivity is substantially ameliorated, without a significant decrease in Seebeck voltage or increase in thermal conductivity, leading to $Z T$ almost tripling in the region of interest. While the thermoelectric figure of merit is still less than bulk, these findings offer a path toward further improving $Z T$ and thus demonstration of an additively manufactured TEG that can match or outperform traditional manufacturing techniques. Optimization of the starting material composition for this purpose, rather than using only off-the-shelf stock, is one promising avenue to decrease resistivity. Furthermore, it is notable that while the billet's $Z T$ saturates at around $350 \mathrm{~K}\left(\sim 80^{\circ} \mathrm{C}\right.$, consistent with vendor and literature reports), the performance of cold-sprayed thermoelectric continues to improve.

Figure 4 shows some of the key properties of a prototype TEG sprayed on the outer surface of a $\mathrm{Cu}$ pipe, using parallel $p$ - and $n$-type $\mathrm{Bi}_{2} \mathrm{Te}_{3}$ electrodes, compared with a similar TEG made by attaching bulk $\mathrm{Bi}_{2} \mathrm{Te}_{3}$ to the pipe using epoxy. The thermal gradient that can be achieved without active cooling is presented in Fig. 4a and is approximately the same for both preparations. At low temperatures $\left(<80^{\circ} \mathrm{C}\right)$, the two TEGs have similar current outputs, but this changes dramatically above $100^{\circ} \mathrm{C}$, with the output of the bulk TEG flattening off and the output of the cold-sprayed TEG continuing a linear increase up to the maximum attainable temperature. This trend is also reflected when output current is plotted as a function of temperature gradient in Fig. 4c, with a linear increase across the full range. The improved performance offered by the cold-sprayed TEG can be understood in two ways. First, the lack of a binding agent fixing it to the pipe, and the excellent mechanical and thermal contact, ensure all heat flows through the thermoelectric, efficiently harvesting the available energy. Second, independent measurements of thermoelectric properties (Fig. 3) demonstrated a plateau in performance for bulk $\mathrm{Bi}_{2} \mathrm{Te}_{3}$ at elevated temperature, but no such limit is observed for cold spray. While this improvement merits further study to ensure direct comparability of bulk and cold spray TEGs, it is immediately clear that such additively manufactured TEGs show great promise.

\section{CONCLUSION}

A new application of cold-spray deposition has been demonstrated that can fabricate bulk pieces of thermoelectric $\mathrm{Bi}_{2} \mathrm{Te}_{3}$ on a wide variety of substrates, without loss of structural integrity. By entraining particles in a supersonic gas flow, millimeter-thick blocks can be built up on flat or curved surfaces in a matter of seconds, providing a pathway to waste heat recovery from narrow pipes or other, more complicated, shapes. The sprayed material is composed of randomly oriented crystallites that match the chemical composition of the precursor billet and is $>99.5 \%$ dense. The Seebeck voltage and thermal conductivity are comparable to or better than bulk $\mathrm{Bi}_{2} \mathrm{Te}_{3}$, while the as-deposited resistivity is an order of magnitude higher. A simple annealing treatment at $400^{\circ} \mathrm{C}$ removes defects and decreases resistivity, increasing $Z T$ to $\sim 0.3$ at operating temperatures of $80^{\circ} \mathrm{C}$. Integrated TEG measurements of $\mathrm{Bi}_{2} \mathrm{Te}_{3}$ sprayed on copper flats or pipes suggest performance comparable to simple devices fabricated from blocks of the bulk material, 
demonstrating that cold spray is a viable alternative to traditional manufacturing approaches for thermoelectric materials.

\section{ACKNOWLEDGEMENTS}

Funding for this project was through the Office of Technology Transition's Technology Commercialization Fund (TCF). Lawrence Livermore National Laboratory is operated by Lawrence Livermore National Security, LLC, for the US Department of Energy, National Nuclear Security Administration under Contract DE-AC52-07NA27344. The ALS is supported by the Director, Office of Science, BES, of DOE under Contract No. DEAC02-05CH11231, DEAC02-06CH11357. Beamline 12.2.2 at the Advanced Light Source is a DOE Office of Science User Facility under Contract No. DE-AC02-05CH11231. Part of this work was performed at GeoSoilEnviroCARS (The University of Chicago, Sector 13), Advanced Photon Source (APS), Argonne National Laboratory. GeoSoilEnviroCARS is supported by the National Science Foundation-Earth Sciences (EAR-1634415) and Department of Energy-GeoSciences (DE-FG02-94ER14466). This research used resources of the Advanced Photon Source, a US Department of Energy (DOE) Office of Science User Facility operated for the DOE Office of Science by Argonne National Laboratory under Contract No. DE-AC02-06CH11357. The authors acknowledge Dr. Joseph M. Zaug for useful discussions and the density measurement using pycnometry.

\section{REFERENCES}

1. F. Gärtner, T. Stoltenhoff, T. Schmidt, and H. Kreye, J. Therm. Spray Technol. 15, 223 (2006).

2. A. Sova, S. Grigoriev, A. Okunkova, and I. Smurov, Int. J. Adv. Manuf. Technol. 69, 2269 (2013).

3. X. Wang, F. Feng, M.A. Klecka, M.D. Mordasky, J.K. Garofano, T. El-Wardany, A. Nardi, and V.K. Champagne, Addit. Manuf. 8, 149 (2015).

4. V.K. Champagne, J. Fail. Anal. Prev. 8, 164 (2008).

5. S. Yin, P. Cavaliere, B. Aldwell, R. Jenkins, H. Liao, W. Li, and R. Lupoi, Addit. Manuf. 21, 628 (2018).
6. T. Schmidt, F. Gärtner, H. Assadi, and H. Kreye, Acta Mater. 54, 729 (2006).

7. M. Hassani-Gangaraj, D. Veysset, V.K. Champagne, K.A. Nelson, and C.A. Schuh, Acta Mater. 158, 430 (2018).

8. H. Assadi, F. Gärtner, T. Klassen, and H. Kreye, Scr. Mater. 162, 512 (2019).

9. L. Ajdelsztajn, J.M. Schoenung, B. Jodoin, and G.E. Kim, Metall. Mater. Trans. A 36, 657 (2005).

10. B. Wölfing, C. Kloc, J. Teubner, and E. Bucher, Phys. Rev. Lett. 86, 4350 (2001)

11. H. Lee, R. Chidambaram Seshadri, S.J. Han, and S. Sampath, Appl. Energy 192, 24 (2017).

12. D.T. Morelli, Springer Handbook of Electronic and Photonic Materials, ed. S. Kasap and P. Capper (Cham: Springer, 2017), p. 1379

13. R. He, G. Schierning, and K. Nielsch, Adv. Mater. Technol. 3, 1700256 (2018)

14. A. Elson, R. Tidball, and A. Hampson, Waste Heat to Power Market Assessment (Berlin: Oak Ridge National Lab. (ORNL), Oak Ridge, TN (United States). Building Technologies Research and Integration Center (BTRIC), 2015).

15. D. Champier, Energy Convers. Manag. 140, 167 (2017).

16. A. El-Desouky, M. Carter, M.A. Andre, P.M. Bardet, and S. LeBlanc, Mater. Lett. 185, 598 (2016).

17. A. El-Desouky, M. Carter, M. Mahmoudi, A. Elwany, and S. LeBlanc, J. Manuf. Process. 25, 411 (2017).

18. R.C. Dykhuizen and M.F. Smith, J. Therm. Spray Technol. 7, 205 (1998).

19. C. Prescher and V.B. Prakapenka, High Press. Res. 3, 223 (2015).

20. W. Kraus and G. Nolze, J. Appl. Crystallogr. 29, 301 (1996).

21. J.D. Roehling, W.L. Smith, T.T. Roehling, B. Vrancken, G.M. Guss, J.T. McKeown, M.R. Hill, and M.J. Matthews, Addit. Manuf. 28, 228 (2019).

22. A.S. Wu, D.W. Brown, M. Kumar, G.F. Gallegos, and W.E. King, Metall. Mater. Trans. A 45, 6260 (2014).

23. T. Mukherjee, W. Zhang, and T. DebRoy, Comput. Mater. Sci. 126, 360 (2017)

24. C. Kittel, Introduction to Solid State Physics, 8th ed. (Hoboken: Wiley, 2005).

25. B. Poudel, Q. Hao, Y. Ma, Y. Lan, A. Minnich, B. Yu, X. Yan, D. Wang, A. Muto, D. Vashaee, X. Chen, J. Liu, M.S. Dresselhaus, G. Chen, and Z. Ren, Science 320, 634 (2008).

26. O. Yamashita, S. Tomiyoshi, and K. Makita, J. Appl. Phys. 93, 368 (2002).

Publisher's Note Springer Nature remains neutral with regard to jurisdictional claims in published maps and institutional affiliations. 